\title{
On the characterization of the solution set for vector equilibrium problem
}

\author{
Gang Wang ${ }^{a, *}$, Lijun Gao \\ a School of Management Science, Qufu Normal University, Shandong Rizhao, 276826, China. \\ ${ }^{b}$ School of Engineering, Qufu Normal University, Shandong, Rizhao, 276826, China.
}

Communicated by Y. J. Cho

\begin{abstract}
In this article, we investigate the nonemptiness and compactness of the solution set for vector equilibrium problem defined in finite-dimensional spaces. We show that vector equilibrium problem has nonempty and compact solution set if and only if linearly scalarized equilibrium problem has nonempty and compact solution set provided that $R_{1}=\{0\}$ holds. Furthermore, we obtain that vector equilibrium problem has nonempty and compact solution set if and only if linearly scalarized equilibrium problem has nonempty and compact solution set when coercivity condition holds. As applications, we employ the obtained results to derive Levitin-Polyak well-posedness, stability analysis and connectedness of the solution set of the vector equilibrium problem. (C) 2017 All rights reserved.
\end{abstract}

Keywords: Vector equilibrium problem, nonemptiness and compactness, asymptotic cone, coercivity condition. 2010 MSC: 90C29, 90C33.

\section{Introduction}

Let $X$ be a finite-dimensional normed space and a nonempty set $K \subseteq X$. Let $Y$ be a real normed space ordered by a proper, closed and convex cone $C$ with int $C \neq \emptyset$, i.e., for $y_{1}, y_{2} \in Y, y_{1} \leqslant c y_{2}$, if and only if $y_{2}-y_{1} \in C$. The vector equilibrium problem, abbreviated as (VEP), is to find $\bar{x} \in K$ such that

$$
h(\bar{x}, y) \notin-\text { int } C, \quad \forall y \in K,
$$

where $h: \mathrm{K} \times \mathrm{K} \rightarrow \mathrm{Y}$ is a vector-valued mapping. For (VEP), a closely related problem is so-called dual vector equilibrium problem, abbreviated as (DVEP), which is to find $\bar{x} \in \mathrm{K}$ such that

$$
\mathrm{h}(\mathrm{y}, \bar{x}) \notin \operatorname{int} \mathrm{C}, \quad \forall \mathrm{y} \in \mathrm{K} .
$$

Throughout this paper, we denote by $\bar{X}$ the solution set for (VEP) and by $\bar{X}^{\mathrm{D}}$ the solution set for (DVEP). It is well-known that the solution set for (VEP) can be characterized by the solution set for (DVEP) under mild conditions [8].

\footnotetext{
${ }^{*}$ Corresponding author

Email addresses: wgglj1977@163.com (Gang Wang), gljwg1977@163.com (Lijun Gao)
} 
(VEP) includes several fundamental mathematical problems, such as vector optimization problem, vector variational inequality problem, Nash equilibrium problem and fixed point problem, which finds applications in economics, finance, image reconstruction, ecology, transportation, network, and elasticity; see [15] and reference therein. One of the most important problems for (VEP) is to investigate the properties of the solution set, such as existence and uniqueness [11-13, 25-27, 31], semicontinuity and sensitivity $[1,7]$, well-posedness $[2,22,28-30,32]$ and connectedness $[16,17,23]$. Among many desirable properties of the solution set, the issue of the nonemptiness and boundedness of the solution set is interesting and important, as it can guarantee the convergence of some solution algorithms (see, e.g., [21, 33, 34]).

Flores-Bazan $[12,13]$ provided characterizations of the nonemptiness and boundedness of the solution set based on asymptotic method for generalized noncoercive equilibrium problem in finite-dimensional spaces and reflexive Banach spaces, respectively. Meanwhile, Bianchi and Pini [6] established similar results by using the coercivity condition for equilibrium problem with pseudomonotone functions, and further extended to quasimonotone functions. Analogous to the scalar case, necessary and/or sufficient conditions on the nonemptiness and boundedness of the solution set for C-psedomonotone (generalized) (VEP) were established in [14] for finite-dimensional spaces and in [3, 11, 25] for reflexive Banach spaces. However, it should be noted that the conditions to characterize the nonemptiness and boundedness of the solution set used in $[3,14,25]$ are relatively strong, which may not hold for vector variational inequalities [18]. To weaken the assumption, Huang et al. [20] gave new characterizations on the nonemptiness and compactness of the solution set for vector variational inequalities via a family of variational inequalities. In this paper, motivated by the works in $[3,11-14,18,20,25]$, we develop some new results to characterize the nonemptiness and compactness of the solution set for (VEP) based on linearly scalarized equilibrium problem. As a by-product, under mild conditions, we show that coercivity condition is weaker than the condition $\mathrm{R}_{1}=\{0\}$.

The rest of the paper is organized as follows: Section 2 is devoted to some basic notations and needed results for our subsequent discussions. In Section 3, under the condition $R_{1}=\{0\}$, we show that (VEP) has nonempty and compact solution set if and only if the linearly scalarized equilibrium problem has nonempty and compact solution set. Furthermore, under coercivity condition, we show that (VEP) has nonempty and compact solution set if and only if the linearly scalarized equilibrium problem has nonempty and compact solution set. In Section 4, we employ the obtained results to derive Levitin-Polyak well-posedness, stability analysis and connectedness of the solution set for (VEP).

\section{Notations and preliminaries}

In this section, we recall some basic notations and preliminary results.

For a real normed space $Y$, denote by $Y^{*}$ the dual space of $Y$. Then for any nonempty subset $\phi$ in $Y$, we denote by $\phi^{*}$ the positive polar cone of $\phi^{*}$, i.e.,

$$
\phi^{*}=\left\{l \in Y^{*}: l(v) \geqslant 0, \forall v \in \phi\right\},
$$

and for closed and convex cone $C$ in $Y$ with int $C \neq \emptyset$, we denote

$$
\mathrm{C}^{* 0}=\left\{\lambda \in \mathrm{C}^{*}: \lambda(e)=1\right\}
$$

for any fixed $e \in$ int $C$. From [20, Lemma 3.4], we know the $C^{* 0}$ is a $w^{*}$-compact base of $C^{*}$.

The following concept plays an important role in characterizations on nonemptiness and compactness of the solution set for (VEP).

Definition 2.1 ([9]). Let $\mathrm{K}$ be a nonempty convex subset of $\mathrm{X}$. The mapping $\mathrm{g}: \mathrm{K} \rightarrow \mathrm{Y}$ is called:

(i) C-convex on $\mathrm{K}$ if

$$
g(\alpha x+(1-\alpha) y) \leqslant c \alpha g(x)+(1-\alpha) g(y), \quad \forall x, y \in K, \quad \alpha \in[0,1] ;
$$

(ii) $\mathrm{C}$-concave on $\mathrm{K}$ if $-\mathrm{g}$ is $\mathrm{C}$-convex; 
(iii) $C$-subconvexlike on $K$, if there exists a $\theta \in \operatorname{int} C$ such that for any $x, y \in K$ and any $\epsilon>0$ and $\alpha \in(0,1)$, there exists $b \in K$ satisfying

$$
g(b) \leqslant c \alpha g(x)+(1-\alpha) g(y)+\epsilon \theta .
$$

One can check that the $\mathrm{C}$-convexity implies the $\mathrm{C}$-subconvexlikeness on $\mathrm{K}$. It is well-known that $\mathrm{g}$ is C-subconvexlike on $\mathrm{K}$ if and only if the set $\mathrm{g}(\mathrm{K})+$ int $\mathrm{C}$ is convex from [9, Prop 1.78].

In order to investigate the relations between (VEP) and linearly scalarized equilibrium problem, we introduce the linearly scalarized equilibrium problem of finding $\bar{x} \in K$

$$
\left(E P_{\lambda}\right) \quad \lambda(h)(\bar{x}, y) \geqslant 0, \quad \forall y \in K,
$$

where $\lambda \in \mathrm{C}^{* 0}$.

For linearly scalarized equilibrium problem, a closely related problem is so-called linearly scalarized dual equilibrium problem, which is to find $\bar{x} \in \mathrm{K}$ such that

$$
\left(\operatorname{DEP}_{\lambda}\right) \quad \lambda(h)(y, \bar{x}) \leqslant 0, \quad \forall y \in K .
$$

Throughout this paper, we denote by $\bar{X}_{\lambda}$ the solution set of $\left(E P_{\lambda}\right)$ and by $\bar{X}_{\lambda}^{\mathrm{D}}$ the solution set of $\left(D E P_{\lambda}\right)$. In what follows, we recall the following monotonicity and pseudomonotonicity.

Definition 2.2 ([5]). The mapping $\mathrm{F}: \mathrm{K} \times \mathrm{K} \rightarrow \mathrm{R}$ is called:

(i) monotone on $K$, if for all $x, y \in K$,

$$
F(x, y)+F(y, x) \leqslant 0
$$

(ii) pseudomonotone on $K$, if for all $x, y \in K$,

$$
F(x, y) \geqslant 0 \Rightarrow F(y, x) \leqslant 0 .
$$

The mapping $\mathrm{h}: \mathrm{K} \times \mathrm{K} \rightarrow \mathrm{Y}$ is called:

(iii) C-monotone on $\mathrm{K}$, if for all $x, y \in K$,

$$
h(x, y)+h(y, x) \in-C
$$

(iv) C-pseudomonotone on $K$, if for all $x, y \in K$,

$$
h(x, y) \notin-\text { int } C \Rightarrow h(y, x) \notin \text { int } C .
$$

Next, we shall give the following lemma to investigate between linearly scalarized (pseudomonotonicity) monotonicity and (C-pseudomonotonicity) C-monotonicity.

Lemma 2.3. Let $\mathrm{h}: \mathrm{K} \times \mathrm{K} \rightarrow \mathrm{Y}$ be a mapping. For all $\lambda \in \mathrm{C}^{* 0}$

(i) if $\mathrm{h}$ is $\mathrm{C}$-monotone on $\mathrm{K}$, then $\lambda(\mathrm{h})$ is monotone and $\lambda(\mathrm{h})$ is pseudomonotone;

(ii) if $\lambda(\mathrm{h})$ is pseudomonotone, then $\mathrm{h}$ is $\mathrm{C}$-pseudomonotone.

Proof.

(i) For $x, y \in K$, if $h$ is $C$-monotone on $K$, then $h(x, y)+h(y, x) \in-C$. So, for all $\lambda \in C^{* 0}$, one has

$$
\lambda(h(x, y)+h(y, x)) \leqslant 0 .
$$

Hence, $\lambda(h)$ is monotone for all $\lambda \in C^{* 0}$. Moreover, $\lambda(h)$ is pseudomonotone for all $\lambda \in C^{* 0}$. 
(ii) For $x, y \in K$, if $\lambda(h)$ is pseudomonotone, then

$$
\lambda(h)(x, y) \geqslant 0 \Rightarrow \lambda(h)(y, x) \leqslant 0 .
$$

If $h(x, y) \notin-$ int $C$, then there exists $\bar{\lambda} \in C^{* 0}$ such that $\bar{\lambda}(h)(x, y) \geqslant 0$. Furthermore $\bar{\lambda}(h)(y, x) \leqslant 0$, since $\bar{\lambda}(h)$ is pseudomonotone. Next, we show by contradiction that

$$
h(y, x) \notin \text { int } C .
$$

If $h(y, x) \in$ int $C$, then for $\bar{\lambda} \in C^{* 0}, \bar{\lambda}(h)(y, x)>0$, which contradicts $\bar{\lambda}(h)(y, x) \leqslant 0$. So, the result holds.

In general, the C-pseudomonotonicity of $h$ does not imply the pseudomonotonicity of $\lambda(h)$ for all $\lambda \in \mathrm{C}^{* 0}$.

Example 2.4. Let $\mathrm{K}=\mathbb{R}_{+}^{1}, \mathrm{C}=\mathbb{R}_{+}^{2}, e=(1,1) \in \operatorname{int} \mathrm{C}, \mathrm{Y}=\mathbb{R}^{2}$ and

$$
h(x, y)=\left(\langle 0, y-x\rangle,\left\langle x^{2}-x, y-x\right\rangle\right) .
$$

It is easy to see that $h(x, y)$ is C-pseudomonotone and

$$
C^{* 0}=\left\{\lambda_{1} \geqslant 0, \lambda_{2} \geqslant 0: \lambda_{1}+\lambda_{2}=1\right\} .
$$

For $\bar{\lambda}=(0,1) \in \mathrm{C}^{* 0}$, we obtain

$$
\bar{\lambda}(h)(x, y)=\left\langle x^{2}-x, y-x\right\rangle .
$$

For $\bar{x}=0$, one has

$$
\bar{\lambda}(h)(\bar{x}, y)=\langle 0, y-0\rangle \geqslant 0, \quad \forall y \in \mathbb{R}_{+} .
$$

However,

$$
\left.\bar{\lambda}(h)(y, \bar{x})=\left\langle y^{2}-y, 0-y\right\rangle>0, \quad \forall y \in\right] 0,1[.
$$

So, $\bar{\lambda}(h)$ is not pseudomonotone.

In order to investigate the nonemptiness and compactness of solution set for (VEP), we introduce definitions and the properties of asymptotic cone and set convergence.

Given any closed set $S \subseteq R^{n}$, we define the asymptotic cone of $S$ as the closed set

$$
S^{\infty}=\left\{d \in R^{n}: t_{k} \rightarrow+\infty, \exists x_{k} \in S, \frac{x_{k}}{t_{k}} \rightarrow d\right\} .
$$

If $S$ is convex, then for given $x_{0} \in K$,

$$
S^{\infty}=\left\{d \in R^{n}: x_{0}+t d \in S, \forall t>0\right\} .
$$

The following notions is from horizon limits of [24]. Let $\left\{S^{v}\right\}$ be a sequence of sets in $X$. The horizon outer limit $\lim \sup _{v}^{\infty} S^{v}$ and the horizon inner limit lim inf $v_{v}^{\infty} S^{v}$ are defined respectively by

$$
\begin{aligned}
& \lim \sup _{v}^{\infty} S^{v}:=\{0\} \bigcup\left\{d \in X: \exists N \in \mathcal{N}_{\infty}^{\sharp}, d^{v} \in S^{v}, \lambda^{v} \searrow 0, \lambda^{v} d^{v} \rightarrow_{N} d\right\}, \\
& \liminf _{\nu}^{\infty} \mathrm{S}^{\nu}:=\{0\} \bigcup\left\{\mathrm{d} \in X: \exists \mathrm{N} \in \mathcal{N}_{\infty}, \mathrm{d}^{\nu} \in \mathrm{S}^{\nu}, \lambda^{\nu} \searrow 0, \lambda^{v} \mathrm{~d}^{\nu} \rightarrow_{\mathrm{N}} \mathrm{d}\right\},
\end{aligned}
$$

where $\mathcal{N}_{\infty}^{\sharp}=\left\{N \in \mathbb{I I N}: \forall N^{\prime} \in \mathcal{N}_{\infty}, N \cap N^{\prime} \neq \emptyset\right\}$ and $\mathcal{N}_{\infty}=\left\{N \in \mathbb{I N}: \forall N^{\prime} \in \mathcal{N}_{\infty}^{\sharp}, N \cap N^{\prime} \neq \emptyset\right\}$.

Lemma 2.5 ([24]). A sequence of sets $\left\{S^{v}\right\}$ in $X$ has the property that $\lim \sup _{v}^{\infty} S^{v}:=\{0\}$ if and only if it is eventually bounded in the sense that for some index set $\mathrm{N} \in \mathcal{N}_{\infty}^{\sharp}$, the set $\bigcup_{v \in N} S^{v}$ is bounded. 

1].

The following lemma is the well-known generalized KKM theorem, we refer the reader to [10, Lemma

Lemma 2.6 ([10]). Let $\mathrm{K} \subseteq \mathrm{X}$ be a nonempty topological vector space $\mathrm{X}$ and $\mathrm{F}: \mathrm{K} \rightarrow 2^{\mathrm{X}}$ be a set-valued mapping from $\mathrm{K}$ into $\mathrm{X}$ satisfying the following properties:

(i) $\mathrm{F}$ is called a generalized KKM mapping: for every finite subset $\mathrm{A}$ of $\mathrm{K}, \operatorname{co}(\mathrm{A}) \subseteq \bigcup_{\mathrm{x} \in \mathrm{A}} \mathrm{F}(\mathrm{x})$, where $\operatorname{co}($. stands for the convex hull;

(ii) $\mathrm{F}(\mathrm{x})$ is closed in $\mathrm{X}$ for every $\mathrm{x} \in \mathrm{K}$;

(iii) $\mathrm{F}\left(\mathrm{x}_{0}\right)$ is compact in $\mathrm{X}$ for some $\mathrm{x}_{0} \in \mathrm{K}$.

Then $\bigcap_{x \in K} F(x) \neq \emptyset$.

\section{Characterizations on nonemptiness and compactness of the solution set for (VEP)}

\subsection{Characterizations with linearly scalarized equilibrium problem}

In this subsection, we shall provide sufficient conditions to guarantee the nonemptiness and compactness of the solution set for (VEP) by linear scalarization. For convenience, we make the following assumptions.

Assumption 3.1. The mapping $\mathrm{h}: \mathrm{K} \times \mathrm{K} \rightarrow \mathrm{Y}$ is such that

(i) $\mathrm{K} \subset \mathrm{X}$ is a nonempty and convex closed set;

(ii) for all $x \in K, h(x, x)=0$ and $h(x,):. K \rightarrow Y$ is C-convex;

(iii) $h(x, y)$ is continuous on $K$.

Before we proceed, technical lemma on the solution set of (VEP) is in order.

Lemma 3.2. For all $x \in \mathrm{K}$, if $\mathrm{h}(\mathrm{x},):. \mathrm{K} \rightarrow \mathrm{Y}$ is $\mathrm{C}$-subconvexlike and $\mathrm{h}(\mathrm{x}, \mathrm{x})=0$, then $\overline{\mathrm{X}}=\bigcup_{\lambda \in \mathrm{C} * 0} \overline{\mathrm{X}}_{\lambda}$.

Proof. For all $\lambda \in \mathrm{C}^{* 0}$ and $\bar{x} \in \overline{\mathrm{X}}_{\lambda}$, then

$$
\lambda(h)(\bar{x}, y) \geqslant 0, \quad \forall y \in K .
$$

We shall prove $\bar{x} \in \bar{X}$ by reductio ad absurdum. Suppose $\bar{x} \notin \bar{X}$, then there exists $y_{0} \in K$ such that

$$
h\left(\bar{x}, y_{0}\right) \in-\text { int } C .
$$

For all $\lambda \in \mathrm{C}^{* 0}$, we have

$$
\lambda(h)\left(\bar{x}, y_{0}\right)<0,
$$

which contradicts (3.1). Consequently, $\bar{x} \in \bar{X}$.

Conversely, if $x \in \bar{X}$, then

$$
h(\bar{x}, y) \notin-\text { int } C, \quad \forall y \in K,
$$

equivalently,

$$
0 \notin \mathrm{h}(\overline{\mathrm{x}}, \mathrm{K})+\text { int } \mathrm{C} .
$$

Since $h(x,$.$) is C-subconvexlike, we deduce h(\bar{x}, K)+$ int $C$ is convex. By the separation theorem of convex sets, there exists $\bar{\lambda} \in Y^{*} \backslash\{0\}$ such that

$$
\bar{\lambda}(h(\bar{x}, y))+\bar{\lambda} c \geqslant 0, \quad \forall y \in K, \quad c \in \text { int } C .
$$


Setting $y=\bar{x}$, from $h(x, x)=0$, we obtain

$$
\bar{\lambda} c \geqslant 0, \quad \forall c \in \text { int } C .
$$

So, $\bar{\lambda} \in C^{*} \backslash\{0\}$. On the other hand, letting $\mathrm{c} \rightarrow 0$ in (3.2), we have

$$
\bar{\lambda}(h(\bar{x}, y)) \geqslant 0, \quad \forall y \in K .
$$

Since $C^{* 0}$ is a $w^{*}$-compact base of $C^{*}$ for $t>0$, there exists $\dot{\lambda} \in C^{* 0}$ with $\dot{\lambda} \neq 0$ such that

$$
\bar{\lambda}=\mathrm{t} \dot{\lambda} \quad \text { and } \dot{\lambda}(h(\bar{x}, y)) \geqslant 0, \quad \forall y \in K
$$

So, $\bar{x} \in \bigcup_{\lambda \in C^{* 0}} \bar{X}_{\lambda}$.

From [5, Proposition 3.1 and Theorem 3.1], we have the following lemma for (VEP).

Lemma 3.3. Assume that Assumption 3.1 holds and $\mathrm{h}$ is $\mathrm{C}-$ pseudomonotone, then $\overline{\mathrm{X}}=\overline{\mathrm{X}}^{\mathrm{D}}$.

By [8, Proposition 3.2 and Remark 3.1], we can obtain the following lemma for linearly scalarized equilibrium problem.

Lemma 3.4. Assume that Assumption 3.1 holds and $\lambda(h)$ is pseudomonotone, $\bar{X}_{\lambda}=\bar{X}_{\lambda}^{\mathrm{D}}$ for all $\lambda \in \mathrm{C}^{* 0}$.

In what follows, we recall from $[12,13]$ the following cone that allows us to obtain characterization for the solution set to the equilibrium problem to be nonempty and compact, which is defined by

$$
\overline{R_{1}}:=\bigcap_{y \in K}\left\{d \in K^{\infty}: h(y, y+t d) \leqslant 0, \forall t>0\right\} .
$$

Furthermore, for (VEP) we have the following definition:

$$
\mathrm{R}_{1}:=\bigcap_{\mathrm{y} \in \mathrm{K}}\left\{\mathrm{d} \in \mathrm{K}^{\infty}: \mathrm{h}(\mathrm{y}, \mathrm{y}+\mathrm{td}) \notin \operatorname{int} \mathrm{C}, \forall \mathrm{t}>0\right\} .
$$

In combination with [13, Theorem 5.8], we have the following lemma to characterize the nonemptiness and compactness of solution set for linearly scalarized equilibrium problem.

Lemma 3.5. Assume that Assumption 3.1 holds for $\lambda \in \mathrm{C}^{* 0}$, and $\lambda(\mathrm{h})$ is pseudomonotone. Then, the solution set $\overline{\mathrm{X}}_{\lambda}$ of the $\left(\mathrm{EP}_{\lambda}\right)$ is nonempty and compact, if and only if

$$
R_{1}^{\lambda}:=\bigcap_{y \in K}\left\{d \in K^{\infty}: \lambda(h)(y, y+t d) \leqslant 0, \forall t>0\right\}=\{0\} .
$$

Theorem 3.6. Assume that Assumption 3.1 holds and $\lambda(h)$ is pseudomonotone for all $\lambda \in C^{* 0}$. If the solution set $\bar{X}_{\lambda}$ of $\left(\mathrm{EP}_{\lambda}\right)$ is nonempty and compact, then $\overline{\mathrm{X}}$ is nonempty and compact.

Proof. Recalling that the solution set $\bar{X}_{\lambda}$ of $\left(E P_{\lambda}\right)$ is nonempty and compact, we obtain that $\bar{X}$ is nonempty. Using the continuity of $h$, we know that $\bar{X}$ is closed. Clearly, $0 \in \bar{X}^{\infty}$. For all $\lambda \in C^{* 0}$, it follows from the nonemptiness and compactness of the solution set for $\left(E P_{\lambda}\right)$ and Lemma 3.5 that

$$
\mathrm{R}_{1}^{\lambda}=\bigcap_{\mathrm{y} \in \mathrm{K}}\left\{\mathrm{d} \in \mathrm{K}^{\infty}: \lambda(\mathrm{h})(\mathrm{y}, \mathrm{y}+\mathrm{td}) \leqslant 0, \forall \mathrm{t}>0\right\}=\{0\} .
$$

Next, we show $\bar{X}^{\infty}=\{0\}$. Suppose that there exists $d \in \bar{X}^{\infty}$ with $d \neq 0$. With the definition of $\bar{X}^{\infty}$, there exist $m_{k} \rightarrow+\infty$ and $x_{k} \in \bar{X}$ with $\left\|x_{k}\right\| \rightarrow \infty$ such that $\frac{x_{k}}{m_{k}} \rightarrow d$. From $x_{k} \in \bar{X}$, there exists $\lambda_{k} \in C^{* 0}$ such that

$$
\lambda_{k}(h)\left(x_{k}, y\right) \geqslant 0
$$


It follows from pseudomonotonicity of the $\lambda(h)$ that

$$
\lambda_{k}(h)\left(y, x_{k}\right) \leqslant 0
$$

Since $C^{* 0}$ is $w^{*}$-compact, without loss of generality, we can assume that

$$
w^{*}-\lim _{k \rightarrow \infty} \lambda_{k}=\lambda^{*} \text {. }
$$

For $\lambda \in \mathrm{C}^{* 0}, \mathrm{y} \in \mathrm{K}$ and $\mathrm{t}>0$, as $\mathrm{m}_{\mathrm{k}} \rightarrow+\infty$ for $\mathrm{k}$ large enough, one has $0<\frac{\mathrm{t}}{\mathrm{m}_{\mathrm{k}}}<1$ and

$$
\lambda_{k}(h)\left(y,\left(1-\frac{t}{m_{k}}\right) y+\frac{t}{m_{k}} x_{k}\right) \leqslant\left(1-\frac{t}{m_{k}}\right) \lambda_{k}(h)(y, y)+\frac{t}{m_{k}} \lambda_{k}(h)\left(y, x_{k}\right) \leqslant 0,
$$

which implies

$$
\lambda_{k}(h)\left(y,\left(1-\frac{t}{m_{k}}\right) y+\frac{t}{m_{k}} x_{k}\right)-\lambda_{k}(h)(y, y+t d)+\left(\lambda_{k}-\lambda^{*}\right)(h)(y, y+t d)+\lambda^{*}(h)(y, y+t d) \leqslant 0 .
$$

Letting $k \rightarrow \infty$ in (3.3), we obtain

$$
\lambda^{*}(h)(y, y+t d) \leqslant 0,
$$

since $h(y, x)$ is continuous and $w^{*}-\lim _{k \rightarrow \infty} \lambda_{k}=\lambda^{*}$. So, $d \in R_{1}^{\lambda^{*}}$ with $d \neq 0$, which contradicts $R_{1}^{\lambda^{*}}=\{0\}$. Thus, $\bar{X}$ is nonempty and compact.

Theorem 3.7. Assume that Assumption 3.1 holds. Then,

(i) $\bigcup_{\lambda \in C^{* 0}} R_{1}^{\lambda} \subseteq R_{1}$.

(ii) If $\lambda(h)$ is pseudomonotone for all $\lambda \in C^{* 0}$ and $R_{1}=\{0\}$, then the solution set $\bar{X}_{\lambda}$ of $\left(\mathrm{EP}_{\lambda}\right)$ is nonempty and compact.

Proof.

(i) For $d \in \bigcup_{\lambda \in C^{* 0}} R_{1}^{\lambda}$, there exists $\lambda \in C^{* 0}$ such that

$$
\lambda(h)(y, y+t d) \leqslant 0, \quad \forall t>0, \quad y \in K .
$$

For all $t>0$, assume by contradiction that $h(y, y+t d) \in \operatorname{int} C$, then

$$
\lambda(h)(y, y+t d)>0, \quad \forall \lambda \in C^{* 0}, \quad y \in K,
$$

which contradicts (3.4). Hence, $\bigcup_{\lambda \in C^{* 0}} R_{1}^{\lambda} \subseteq R_{1}$.

(ii) It follows from $0 \in R_{1}^{\lambda} \subseteq \bigcup_{\lambda \in C^{* 0} 0} R_{1}^{\lambda} \subseteq R_{1}=\{0\}$ that $R_{1}^{\lambda}=\{0\}$. From Theorem 3.6, we deduce that the solution set $\bar{X}_{\lambda}$ of $\left(E P_{\lambda}\right)$ is nonempty and compact.

Remark 3.8. From Theorem 3.7, we note that $R_{1}=\{0\}$ implies $\bigcup_{\lambda \in C_{* 0}} R_{1}^{\lambda}=\{0\}$, which shows that $\bigcup_{\lambda \in C * 0} R_{1}^{\lambda}=\{0\}$ is weak condition for characterizing the solution set to be nonempty and compact for (VEP) and (DVEP). It is worth noting that the solution set for (VEP) cannot be characterized by the solution set of finite $\left(E P_{\lambda}\right)$ even if $Y=\mathbb{R}^{n}$ and $C=\mathbb{R}_{+}^{n}$. The counter example can be found in [20, Example 3.1].

The following example shows that the inclusion $\bigcup_{\lambda \in C^{* 0}} R_{1}^{\lambda} \subset R_{1}$.

Example 3.9. Let $\mathrm{K}=\mathbb{R}_{+}^{1}, \mathrm{C}=\mathbb{R}_{+}^{2}, \mathrm{Y}=\mathbb{R}^{2}, e=(1,1) \in$ int $\mathrm{C}$ and

It is clear that

$$
h(y, x)= \begin{cases}\left(0,(1-y)\left(x^{2}-y^{2}\right)\right), & 0 \leqslant y \leqslant 1 \\ \left((y-1)\left(x^{2}-y^{2}\right), 0\right), & y>1\end{cases}
$$

$$
\mathrm{C}^{* 0}=\left\{\left(\lambda_{1}, \lambda_{2}\right): \lambda_{1}+\lambda_{2}=1, \lambda_{1} \geqslant 0, \lambda_{2} \geqslant 0\right\} .
$$

For all $y \in K$, it is verified that

$$
\bigcup_{\lambda \in C^{* 0}} R_{1}^{\lambda}=\{0\}
$$

On the other hand, by simple computation, we obtain $R_{1}=[0,+\infty)$. So, $\bigcup_{\lambda \in C^{* 0}} R_{1}^{\lambda} \subset R_{1}$. 
Corollary 3.10. Assume that Assumption 3.1 holds, $\lambda(\mathrm{h})$ is pseudomonotone for all $\lambda \in \mathrm{C}^{* 0}$ and $\mathrm{R}_{1}=\{0\}$. Then, the solution set $\bar{X}_{\lambda}$ of $\left(\mathrm{EP}_{\lambda}\right)$ is nonempty and compact if and only if that the solution set $\overline{\mathrm{X}}$ for $(V E P)$ is nonempty and compact.

\subsection{Characterizations with coercivity condition}

The purpose of the subsection is to show that coercivity condition is a sufficient condition to guarantee the nonemptiness and compactness of the solution set for (VEP) when $K$ is unbounded. Meanwhile, it is worth noting that the nonemptiness and compactness of $\bar{X}$ cannot imply that $\bar{X}_{\lambda}$ is nonempty for all $\lambda \in \mathrm{C}^{* 0}$.

For convenience, $K_{r}$ is defined by

$$
K_{r}:=\{x: x \in K,\|x\| \leqslant r\}
$$

Then, $K_{r}$ is a compact subset of $K$.

Consider the following coercivity condition [5]:

(C)

$$
\exists r>0: \forall x \in K \backslash K_{r}, \exists y \in K_{r} \text { such that } h(x, y) \in-\text { int } C \text {. }
$$

The following conclusion may be not new. However, we include a compact stand-alone proof below, both for the sake of completeness and because the proof is used in proving our next result.

Theorem 3.11. If Assumption 3.1 holds and coercivity condition (C) is satisfied, then the solution set $\bar{X}$ is nonempty and compact.

Proof. Define $\mathrm{H}: \mathrm{K} \times \mathrm{K} \rightarrow 2^{\mathrm{Y}}$ by

$$
\mathrm{H}(\mathrm{y})=\{x \in \mathrm{K}: \mathrm{h}(x, y) \notin-\text { int } C\},
$$

for each $y \in K$. To show the assertion, we need to verify that $H$ is a generalized KKM mapping. Suppose there exists a finite set $\left\{y_{1}, \ldots, y_{m}\right\} \in K$ such that $x \in \operatorname{co}\left\{y_{1}, \cdots, y_{m}\right\}$ with $x \notin H\left(y_{i}\right)$, for all $i=1, \cdots, m$. Note that

$$
h\left(x, y_{i}\right) \in-\operatorname{int} C, \quad i=1,2, \cdots, m .
$$

Moreover,

$$
\sum_{i=1}^{m} t_{i} h\left(x, y_{i}\right) \in-\text { int } C
$$

The C-convexity of $h$ implies

$$
\sum_{i=1}^{m} t_{i} h\left(x, y_{i}\right) \in h(x, x)+C \subset Y \backslash-\text { int } C,
$$

which contradicts (3.5). So, $\mathrm{H}(\mathrm{y})$ satisfies the finite-intersection property. This, in combination with Assumption 3.1, provides that $\mathrm{H}$ is a generalized KKM mapping with closed values. Since the coercivity condition $(C)$ holds with $r>0$, one derives $\bigcap_{y \in K} H(y) \subseteq K_{r}$. It follows from Lemma 2.6 that $\bigcap_{y \in K} H(y) \neq$ $\emptyset$ and $\bar{X} \neq \emptyset$. From $\bar{X} \subseteq K_{r}$, we obtain that the solution set for (VEP) is nonempty and compact.

Example 3.12. Let $\mathrm{K}=\mathbb{R}_{-}^{1}, \mathrm{C}=\mathbb{R}_{+}^{2}, \mathrm{Y}=\mathbb{R}^{2}, \mathrm{e}=(1,1) \in$ int $\mathrm{C}$ and

$$
h(x, y)=\left(\left\langle x^{2}, y-x\right\rangle,\langle 1, y-x\rangle\right) .
$$

It is clear that Assumption 3.1 holds, $h(x, y)$ is C-convex and

$$
C^{* 0}=\left\{\left(\lambda_{1}, \lambda_{2}\right): \lambda_{1}+\lambda_{2}=1, \lambda_{1} \geqslant 0, \lambda_{2} \geqslant 0\right\} .
$$

We break up the proof into two cases to verify that $\bar{X}=\{0\}$. 
(i) For the vector $\bar{\lambda}=(1,0) \in \mathrm{C}^{* 0}$ and $\bar{x}=0$, then

$$
\bar{\lambda}(h(\bar{x}, y))=\langle 0, y-0\rangle \geqslant 0, \quad \forall y \in K .
$$

So, $\operatorname{EP}_{\bar{\lambda}}=\{0\}$.

(ii) For vector $C=(t, 1-t) \in C^{* 0}, t \in[0,1[$, then

$$
\lambda(h(x, y))=\left\langle t x^{2}+(1-t), y-x\right\rangle .
$$

For $x \in K$, there exists $\tilde{y}$ such that

$$
\lambda(h(x, \tilde{y}))=\left\langle t x^{2}+(1-t), \tilde{y}-x\right\rangle<0 .
$$

Hence, for $\lambda=(t, 1-t) \in C^{* 0}$ with $t \in\left[0,1\left[,\left(E P_{\lambda}\right)=\emptyset\right.\right.$. From Lemma 3.2, we know that

$$
\overline{\mathrm{X}}=\bigcup_{\lambda \in \mathrm{C}^{* 0}} \overline{\mathrm{X}}_{\lambda}=\{0\},
$$

is nonempty and compact. However, for all $x \neq 0$ and $|y| \leqslant|x|$, one has

$$
h(x, y) \notin-\text { int } C \text {. }
$$

Consequently, coercivity condition (C) does not hold.

Remark 3.13.

(i) The nonemptiness and compactness of solution set for (VEP) cannot deduce that coercivity condition (C) holds by Example 3.12.

(ii) From Example 3.12, the nonemptiness and compactness of $\bar{X}$ cannot imply that $\bar{X}_{\lambda}$ is nonempty for all $\lambda \in \mathrm{C}^{* 0}$.

Corollary 3.14. If Assumption 3.1 holds and coercivity condition (C) is satisfied, then the solution set for $\left(\mathrm{EP}_{\lambda}\right)$ is nonempty and compact for all $\lambda \in \mathrm{C}^{* 0}$.

Proof. Since coercivity condition (C) holds, there exists $r>0$ such that for all $x \in K \backslash K_{r}$, there exists $y \in K_{r}$,

$$
\lambda(h)(x, y)<0, \quad \forall \lambda \in C^{* 0} .
$$

Set

$$
H_{\lambda}(y):=\{x \in K: \lambda(h)(x, y) \geqslant 0\},
$$

for each $y \in K$. Following the similar arguments in the proof of Theorem 3.11, we can obtain the desired result.

Corollary 3.15. Assume that Assumption 3.1 holds and coercivity condition $(C)$ is satisfied. Then, for all $\lambda \in \mathrm{C}^{* 0}$, the solution set $\bar{X}_{\lambda}$ of $\left(\mathrm{EP}_{\lambda}\right)$ is nonempty and compact if and only if the solution set $\overline{\mathrm{X}}$ of $(V E P)$ is nonempty and compact.

3.3. Comparison between coercivity condition $(C)$ and asymptotic cone $R_{1}=\{0\}$

In this subsection, we shall discuss relations between coercivity condition $(C)$ and $R_{1}=\{0\}$, since coercivity condition $(C)$ and $R_{1}=\{0\}$ play a conspicuous part in characterizing the nonemptiness and compactness of the solution set for (VEP).

Theorem 3.16. Assume that Assumption 3.1 holds and the mapping $\mathrm{h}$ is $\mathrm{C}$-pseudomonotone. If $\mathrm{R}_{1}=\{0\}$, then coercivity condition (C) holds. 
Proof. Suppose that coercivity condition (C) fails. For every $r>0$, there exists $x_{r} \in K \backslash K_{r}$ such that

$$
h\left(x_{r}, y\right) \in Y-\operatorname{int} C, \quad \forall y \in K_{r} .
$$

Set

$$
\digamma_{\mathrm{r}}:=\left\{x_{\mathrm{r}} \in K \backslash K_{\mathrm{r}}: h\left(x_{\mathrm{r}}, y\right) \in Y \backslash \text { int } C, \forall y \in K_{\mathrm{r}}\right\},
$$

and

$$
S_{\mathrm{r}}:=\left\{\bar{x} \in \mathrm{K}: \mathrm{h}(\overline{\mathrm{x}}, \mathrm{y}) \in \mathrm{Y} \backslash-\text { int } \mathrm{C}, \forall \mathrm{y} \in \mathrm{K}_{\mathrm{r}}\right\} .
$$

It is easy to see that $\digamma_{\mathrm{r}} \subseteq S_{\mathrm{r}}$ and $\digamma_{\mathrm{r}}$ is unbounded as $r \rightarrow \infty$. Next, we claim

$$
\lim \sup _{\mathrm{r} \rightarrow \infty}^{\infty} S_{\mathrm{r}}=\{0\} .
$$

Suppose to the contrary that there exists $d \in \limsup _{r \rightarrow \infty}^{\infty} S_{r}$ with $d \neq 0$. Then, there exist $r_{k} \rightarrow \infty, x_{r} \in S_{r_{k}}$ and $m_{k} \rightarrow+\infty$ such that $\frac{x_{k}}{r_{k}} \rightarrow d$. Clearly, $d \in K^{\infty}$ and $\left\|x_{k}\right\| \rightarrow \infty$ as $k \rightarrow \infty$. Then for any fixed $y \in K$, from $x_{k} \in S_{r_{k}}$, we know

$$
h\left(x_{k}, y\right) \in Y-\text { int } C, \quad \forall y \in K_{r}
$$

for all sufficiently large $k$. By the $C$-pseudomonotone of $h$, we have

$$
h\left(y, x_{k}\right) \in Y \backslash \text { int } C, \quad \forall y \in K_{r}
$$

for all sufficiently large $k$. For $y \in K$ and $t>0$, as $r_{k} \rightarrow+\infty$ for $k$ large enough, one has $0<\frac{t}{r_{k}}<1$ and

$$
h\left(y,\left(1-\frac{t}{r_{k}}\right) y+\frac{t}{r_{k}} x_{k}\right) \in\left(1-\frac{t}{r_{k}}\right) h(y, y)+\frac{t}{r_{k}} h\left(y, x_{k}\right)-C,
$$

since $h(x,$.$) is C-convex. It follows from h(y, y)=0$ and $h\left(y, x_{k}\right) \in Y \backslash$ int $C$ that

$$
h\left(y,\left(1-\frac{t}{r_{k}}\right) y+\frac{t}{r_{k}} x_{k}\right) \in Y \backslash \text { int } C-C \subseteq Y \backslash \text { int } C .
$$

Letting $k \rightarrow \infty$ in (3.6), we deduce

$$
h(y, y+t d) \in Y \backslash \text { int } C,
$$

which implies $d \in R_{1}=\{0\}$. This contradicts the $d \neq 0$. Hence,

$$
\lim \sup _{r \rightarrow \infty}^{\infty} S_{r}=\{0\} .
$$

As (3.7) holds, it follows from Lemma 2.5 that $S_{r}$ is eventually bounded. This shows that $\bar{X}$ is bounded and there exists $r_{0}>0$ such that $S_{r}$ is nonempty and compact for all $r>r_{0}$. Since $\digamma_{r} \subseteq S_{r}$, this means that $\digamma_{\mathrm{r}}$ is nonempty and compact for all $r>r_{0}$, which contradicts that $\digamma_{\mathrm{r}}$ is unbounded as $r \rightarrow \infty$. So, coercivity condition (C) holds.

As a consequence of Lemma 2.3, Theorem 3.6, Remark 3.8, Theorem 3.7 and Theorem 3.11, we have the following result.

Theorem 3.17. Assume that Assumption 3.1 holds and the mapping $\mathrm{h}$ is $\mathrm{C}$-pseudomonotone. Consider the following statements:

(i) $R_{1}=\{0\}$;

(ii) the coercivity condition (C) holds;

(iii) the solution set $\bar{X}_{\lambda}$ of $\left(\mathrm{EP}_{\lambda}\right)$ is nonempty and compact;

(iv) the solution set of (VEP) is nonempty and compact.

Then, (i) $\Rightarrow$ (ii) $\Rightarrow$ (iii) and (ii) $\Rightarrow$ (iv); if $\lambda\left(\mathrm{h}\right.$ ) is pseudomonotone, then, (i) $\Rightarrow$ (ii) $\Rightarrow$ (iii) $\Rightarrow$ (iv). If $\overline{\mathrm{X}}_{\lambda} \neq \emptyset$ for all $\lambda \in \mathrm{C}^{* 0}$, then (iv) $\Rightarrow$ (iii).

Remark 3.18. Compared with [20, Theorem 3.3], Theorem 3.17 establishes weaker condition to obtain the equivalence between the nonemptiness and compactness of the solution set for (VEP) and the nonemptiness and compactness of the solution set for $\left(E P_{\lambda}\right)$, since Theorem 3.16 and Corollary 3.15 hold. 


\section{Applications}

\subsection{Levitin-Polyak well-posedness for (VEP)}

Levitin-Polyak well-posedness acts as a crucial role in numerical algorithms theory for (VEP), as it guarantees that, for an approximating solution sequence, there exists a subsequence that converges to a solution. In this subsection, we establish the equivalence between the nonemptiness and compactness of the solution set for (VEP) and Levitin-Polyak well-posedness for (VEP) under mild conditions, which generalizes and extends some results of $[2,22,28,30,32]$ in some sense.

Denote by $d_{K}(x)$ the distance function from a point $x$ to set $K$. We introduce the following definition of Levitin-Polyak well-posed property for (VEP).

\section{Definition 4.1.}

(i) A sequence $\left\{x_{n}\right\} \subseteq X$ is called a Levitin-Polyak approximating solution sequence if there exists a sequence $\left\{\epsilon_{\mathrm{n}}\right\} \subseteq \mathrm{R}_{+}^{1}$ with $\epsilon_{\mathrm{n}} \rightarrow 0$ such that

$$
\begin{gathered}
d_{K}\left(x_{n}\right) \leqslant \epsilon_{n}, \\
h\left(x_{n}, y\right)+\epsilon_{n} e \notin-\operatorname{int} C, \quad \forall y \in K .
\end{gathered}
$$

(ii) (VEP) is called Levitin-Polyak well-posed if the solution set $\bar{X}$ for (VEP) is nonempty, and for LevitinPolyak approximating solution sequence $\left\{x_{n}\right\}$, there exist a subsequence $\left\{x_{n_{j}}\right\}$ of $\left\{x_{n}\right\}$ and $\bar{x} \in \bar{X}$ such that $x_{n_{j}} \rightarrow \bar{x}$.

Theorem 4.2. Assume that Assumption 3.1 holds. If $\mathrm{R}_{1}=\{0\}$ and $\mathrm{h}$ is $C$-monotone, then the solution set $\overline{\mathrm{X}}$ of the $(V E P)$ is nonempty and compact if and only if $(V E P)$ is Levitin-Polyak well-posed.

Proof. On one hand, it follows from the definition of Levitin-Polyak well-posedness that the solution set $\overline{\mathrm{X}}$ of (VEP) is nonempty and compact.

On the other hand, let $\left\{x_{n}\right\} \subset X$ be a Levitin-Polyak approximating solution sequence satisfying (4.1) and (4.2). It follows from Theorem 3.17 that $\left(E P_{\lambda}\right)$ is nonempty and compact for all $\lambda \in C^{* 0}$. Now, we show that $\left\{x_{n}\right\}$ is bounded. Suppose to the contrary that $\left\{x_{n}\right\}$ is unbounded. We assume without loss of generality that $\left\|x_{n}\right\| \rightarrow+\infty$ such that

$$
h\left(x_{n}, y\right)+\epsilon_{n} e \notin-\text { int } C, \quad \forall y \in K,
$$

namely,

$$
h\left(x_{n}, y\right) \bigcap\left(-\operatorname{int} C-\epsilon_{n} e\right)=\emptyset, \quad \forall y \in K .
$$

Since $h(x,$.$) is C-convex, one has h\left(x_{n}, K\right)$ is a convex set and $\left(-\right.$ int $\left.C-\epsilon_{n} e\right)$ is a convex set. By the separation theorem of convex sets, there exists $\lambda_{n} \in C^{* 0}$ such that

$$
\lambda_{\mathrm{n}}(\mathrm{h})\left(x_{\mathrm{n}}, \mathrm{y}\right) \geqslant-\epsilon_{\mathrm{n}}, \quad \forall \mathrm{y} \in \mathrm{K} .
$$

Since $C^{* 0}$ is $\omega^{*}$-compact, we can assume without loss of generality that

$$
\omega^{*}-\lim _{n \rightarrow+\infty} \lambda_{n}=\bar{\lambda} \in C^{* 0} .
$$

In view of $\mathrm{C}$-monotonicity of $\mathrm{h}(\mathrm{x}, \mathrm{y})$ on $\mathrm{K}$, we have

$$
h\left(x_{n}, y\right)+h\left(y, x_{n}\right) \in-C,
$$

that is,

$$
h\left(x_{n}, y\right)+\epsilon_{n} e+h\left(y, x_{n}\right)-\epsilon_{n} e \in-C,
$$




$$
\lambda\left(h\left(x_{n}, y\right)+\epsilon_{n} e+h\left(y, x_{n}\right)-\epsilon_{n} e\right) \leqslant 0, \quad \lambda \in C^{* 0} .
$$

For $\lambda_{\mathrm{n}} \in \mathrm{C}^{* 0}$, one has

$$
\lambda_{\mathrm{n}}(\mathrm{h})\left(\mathrm{y}, \mathrm{x}_{\mathrm{n}}\right) \leqslant \epsilon_{\mathrm{n}}, \quad \forall \mathrm{y} \in \mathrm{K} .
$$

Let $y \in K$ be an arbitrary point. We can assume without loss of generality that

$$
\lim _{n \rightarrow+\infty} \frac{x_{n}}{\left\|x_{n}\right\|}=\lim _{n \rightarrow+\infty} \frac{x_{n}-y}{\left\|x_{n}-y\right\|}=d \text {. }
$$

Thus, $\|\mathrm{d}\|=1$ and $\mathrm{d} \in \mathrm{K}^{\infty}$ with $\mathrm{d} \neq 0$. For given $\mathrm{y} \in \mathrm{K}$ and $\mathrm{t}>0$, as $\left\|x_{n}\right\| \rightarrow+\infty$ for $n$ large enough, one has $0<\frac{t}{\left\|x_{n}-y\right\|}<1$ and

$$
\lambda_{n}(h)\left(y, y+t \frac{x_{n}-y}{\left\|x_{n}-y\right\|}\right) \leqslant \frac{\left\|x_{n}-y\right\|-t}{\left\|x_{n}-y\right\|} \lambda_{n}(h)(y, y)+\frac{t}{\left\|x_{n}-y\right\|} \lambda_{n}(h)\left(y, x_{n}\right),
$$

which is equivalent to

$$
\lambda_{n}(h)\left(y, y+t \frac{x_{n}-y}{\left\|x_{n}-y\right\|}\right) \leqslant \frac{t}{\left\|x_{n}-y\right\|} \lambda_{n}(h)\left(y, x_{n}\right) \leqslant \epsilon_{n},
$$

since $\lambda_{n}(h)$ is convex, $h(y, y)=0$ and $\lambda_{n}(h)\left(y, x_{n}\right) \leqslant \epsilon_{n}$. That is,

$$
\lambda_{n}(h)\left(y, y+t \frac{x_{n}-y}{\left\|x_{n}-y\right\|}\right)-\lambda_{n}(h)(y, y+t d)+\left(\lambda_{n}-\bar{\lambda}\right)(h)(y, y+t d)+\bar{\lambda}(h)(y, y+t d) \leqslant \epsilon_{n} .
$$

For (4.3), taking the limit in k, one has

$$
\bar{\lambda}(h(y, y+t d)) \leqslant 0,
$$

since $h(y, x)$ is continuous and $w^{*}-\lim _{k \rightarrow \infty} \lambda_{k}=\lambda^{*}$. That is, $0 \neq d \in R_{\bar{\lambda}}$, contradicting the fact that the solution set of $E P_{\bar{\lambda}}$ is nonempty and compact. Consequently, $\left\{x_{n}\right\}$ is bounded. There exist a subsequence $\left\{x_{n_{j}}\right\}$ of $\left\{x_{n}\right\}$ and $\bar{x}$ such that $x_{n_{j}} \rightarrow \bar{x}$. Taking the limit in (4.1) (with $n$ replaced by $n_{j}$ ), we have $\bar{x} \in K$. Moreover, taking the limit in (4.2) (with $n$ replaced by $n_{j}$ ), we obtain $h(\bar{x}, y) \notin-$ int $C$, for all $y \in K$. So $\bar{\chi} \in \bar{X}$.

Remark 4.3. Compared with [22, Theorem 4.1], Theorem 4.2 proposes linear scalarization method to obtain Levitin-Polyak well-posedness for (VEP). Under condition $R_{1}=\{0\}$, we obtain that the solution set $\bar{X}$ of (VEP) is nonempty and compact if and only if (VEP) is Levitin-Polyak well-posed. Although the condition is relatively strong, it is the first time to obtain the equivalence between the nonemptiness and compactness of the solution set and Levitin-Polyak well-posedness of (VEP).

\subsection{Stability analysis for (VEP)}

The stability of the solution set is an important problem in the theory of equilibrium problem, as they can guarantee solution set continuity $[10,19,27]$. In this subsection, we shall establish the stability theorems for (VEP) and (DVEP) when the mapping $h$ is perturbed.

Firstly, we recall some important notions and results. Let $h: Z \times K \times K \rightarrow Y$ be a parametric vectorvalued mapping. Consider the perturbed vector equilibrium problem, abbreviated as (PVEP), is to find $\bar{x} \in \mathrm{K}$ such that

$$
h(z, \bar{x}, y) \notin-\text { int } C, \quad \forall y \in K .
$$

For (PVEP), a closely related problem is so-called perturbed dual vector equilibrium problem, abbreviated as (PDVEP), which is to find $\bar{x} \in K$ such that

$$
h(z, y, \bar{x}) \notin \text { int } C, \quad \forall y \in K .
$$

We denote by $\bar{X}(z)$ the solution set of the (PVEP) and by $\bar{X}^{\mathrm{D}}(z)$ the solution set of the (PDVEP). Similar to the previous linear scalarization method, we denote by $\bar{X}_{\lambda}(z)$ the solution set of $\left(P E P_{\lambda}\right)$ and by $\bar{X}_{\lambda}^{\mathrm{D}}(z)$ the solution set of $\left(\mathrm{PDEP}_{\lambda}\right)$. 
Theorem 4.4. Assume that $\mathrm{h}(z, \mathrm{x},):. \mathrm{K} \rightarrow \mathrm{Y}$ is C-convex for all $(z, \mathrm{x}) \in \mathrm{Z} \times \mathrm{K}, \mathrm{h}(z, x, \mathrm{x})=0$ and $\mathrm{h}(z, \mathrm{x}, \mathrm{y})$ is continuous on $\mathrm{Z} \times \mathrm{K} \times \mathrm{K}$. Let $\lambda(\mathrm{h})$ be pseudomonotone for all $\lambda \in \mathrm{C}^{* 0}$. If the solution set $\overline{\mathrm{X}}_{\lambda}\left(z_{0}\right)$ of $\left(\mathrm{PEP}_{\lambda}\right)$ is nonempty and compact for all $\lambda \in \mathrm{C}^{* 0}$, then there exists a neighborhood $\mathrm{U}$ of $z_{0}$ such that (PVEP) and (PDVEP) have a nonempty and compact solution set for all $z \in \mathrm{U}$.

Proof. Since $\bar{X}_{\lambda}\left(z_{0}\right)$ is nonempty and compact and $\lambda(h)$ is pseudomonotone, it follows from Lemma 3.4 that $\bar{X}_{\lambda}^{\mathrm{D}}\left(z_{0}\right)$ is nonempty and compact and

$$
\mathrm{R}_{1}^{\lambda}\left(z_{0}\right)=\bigcap_{y \in K}\left\{\mathrm{~d} \in \mathrm{K}^{\infty}: \lambda(\mathrm{h})\left(z_{0}, \mathrm{y}, \mathrm{y}+\mathrm{td}\right) \leqslant 0, \forall \mathrm{t}>0\right\}=\{0\} .
$$

We claim that there exists a neighborhood $\mathrm{U}_{\lambda}$ of $z_{0}$ such that

$$
\mathrm{R}_{1}^{\lambda}(z)=\bigcap_{y \in K}\left\{\mathrm{~d} \in \mathrm{K}^{\infty}: \lambda(\mathrm{h})(z, y, y+\mathrm{td}) \leqslant 0, \forall \mathrm{t}>0\right\}=\{0\} .
$$

Assume by contradiction that there exists $z_{\mathfrak{n}} \rightarrow z_{0}$ such that $R_{1}^{\lambda}(z) \neq\{0\}$. Thus, we can select a sequence $d_{n}$ such that

$$
\bigcap_{y \in K}\left\{d_{n} \in K^{\infty}: \lambda(h)\left(z_{n}, y, y+t d_{n}\right) \leqslant 0, \forall t>0\right\},
$$

with $\left\|d_{n}\right\|=1$. Without loss of generality, we can assume $d_{\mathfrak{n}} \rightarrow d_{0} \neq 0$. Taking the limit in (4.4), we have

$$
\bigcap_{y \in K}\left\{d_{0} \in K^{\infty}: \lambda(h)\left(z_{0}, y, y+t_{0}\right) \leqslant 0, \forall t>0\right\},
$$

since $h$ is continuous and $\lambda(h)$ is continuous. This contradicts $R_{1}^{\lambda}\left(z_{0}\right)=\{0\}$. Set $\bigcap_{\lambda \in C^{* 0}} U_{\lambda}=U \neq \emptyset$. Hence, there exists a neighborhood $U$ of $z_{0}$ such that $R_{1}^{\lambda}(z)=\{0\}$ for all $\lambda \in C^{* 0}$. It follows from Theorem 3.16 and Lemma 3.5 that (PVEP) and (PDVEP) have a nonempty and compact solution set for all $z \in \mathrm{U}$.

\subsection{Connectedness of the solution set for (VEP)}

The purpose of this subsection is to discuss the connectedness of the solution set for (VEP), as it provides the possibility of continuously moving from one solution to any other solution. We propose new sufficient conditions to establish the connectedness of the solution set for (VEP) without the compactness or monotonicity, which generalizes and extends some results of $[16,17,23]$ in some sense.

Lemma 4.5 ([4]). Let $\mathrm{X}$ and $\mathrm{Y}$ be two topological vector spaces, $\mathrm{S}$ be a connected subset of $\mathrm{X}, \mathrm{F}: \mathrm{S} \rightarrow 2^{\mathrm{Y}}$ be a set-valued mapping. If $\mathrm{F}$ is upper semicontinuous on $\mathrm{S}$ and $\mathrm{F}(\mathrm{x})$ is connected subset of $\mathrm{Y}$ for each $\mathrm{x} \in \mathrm{S}$, then $\mathrm{F}(\mathrm{S})=\bigcup_{\mathrm{x} \in \mathrm{S}} \mathrm{F}(\mathrm{x})$ is a connected subset of $\mathrm{Y}$.

Theorem 4.6. Assume that Assumption 3.1 holds and coercivity condition $(C)$ is satisfied. Let $\mathrm{h}(., \mathrm{y})$ be $\mathrm{C}$-concave on $\mathrm{K}$. If $\mathrm{W}=\{\mathrm{h}(\mathrm{x}, \mathrm{y}): \mathrm{x}, \mathrm{y} \in \mathrm{K}\}$ is bounded, then the solution set for (VEP) is connected.

Proof. It follows from Corollary 3.14 that the solution set $\bar{X}_{\lambda}$ of $\left(E P_{\lambda}\right)$ is nonempty and compact for all $\lambda \in C^{* 0}$. Indeed, for any $\lambda \in C^{* 0}$ and any fixed $y \in K$, let $x_{1}, x_{2} \in \bar{X}_{\lambda}$ and $t \in[0,1]$. Then, $t x_{1}+(1-t) x_{2} \in K$, $\lambda(h)\left(x_{1}, y\right) \geqslant 0$ and $\lambda(h)\left(x_{2}, y\right) \geqslant 0$. From the C-concavity of $h(., y)$ and $\lambda \in C^{* 0}$, we have

$$
\lambda(h)\left(t x_{1}+(1-t) x_{2}, y\right) \geqslant 0,
$$

which shows $t x_{1}+(1-t) x_{2} \in \bar{X}_{\lambda}$. So, $\bar{X}_{\lambda}$ is convex and connected.

Define a set-valued mapping $\mathrm{L}: \mathrm{C}^{* 0} \rightarrow 2^{\mathrm{K}}$ by

$$
\mathrm{L}(\lambda)=\overline{\mathrm{X}}_{\lambda}, \quad \forall \lambda \in \mathrm{C}^{* 0}
$$

Now we show that $L(\lambda)$ is upper semicontinuous on $C^{* 0}$. Since coercivity condition $(C)$ is satisfied, then 
$L\left(\lambda_{n}\right)$ is bounded. We need only prove that $L$ is closed. Let $\left\{\left(\lambda_{n}, x_{n}\right)\right\}$ be a solution sequence such that

$$
\left(\lambda_{n}, x_{n}\right) \subset \operatorname{Graph}(L)=\left\{(\lambda, x) \in C^{* 0} \times K: x \in L(\lambda)\right\},
$$

that is,

$$
\lambda_{\mathrm{n}}(\mathrm{h})\left(\mathrm{x}_{\mathrm{n}}, \mathrm{y}\right) \geqslant 0, \quad \forall \mathrm{y} \in \mathrm{K} .
$$

Since $C^{* 0}$ is $\omega^{*}$-compact, without loss of generality, we can assume that

$$
\omega^{*}-\lim _{n \rightarrow+\infty} \lambda_{n}=\bar{\lambda} \in C^{* 0} .
$$

It follows from the definition of $L(\lambda)$ and coercivity condition (C) that $\left\{x_{n}\right\}$ is bounded. Without loss of generality, we assume $x_{n} \rightarrow \bar{x}$. By the boundedness of $W$ and $\lim _{n \rightarrow+\infty} \lambda_{n}=\bar{\lambda}$, for $\epsilon>0$, there exists $n_{0}$ such that

$$
\left|\left(\lambda_{\mathrm{n}}-\bar{\lambda}\right)(\mathrm{h})\left(x_{\mathrm{n}}, \mathrm{y}\right)\right|<\epsilon, \quad \forall \mathrm{n}>\mathrm{n}_{0} .
$$

So,

$$
\lambda(h)\left(x_{n}, y\right)=\left(\lambda_{n}-\bar{\lambda}\right)(h)\left(x_{n}, y\right)+\bar{\lambda}(h)\left(x_{n}, y\right) \geqslant 0, \quad \forall y \in K \text { and } n>n_{0},
$$

furthermore,

$$
\bar{\lambda}(h)\left(x_{n}, y\right) \geqslant-\epsilon, \quad \forall y \in K \text { and } \mathrm{n}>\mathrm{n}_{0} .
$$

Taking the limit in (4.5), we have $\bar{\lambda}(h)(\bar{x}, y) \geqslant 0$ by the continuity of $h$ and the arbitrariness of $\epsilon$. That is, $\bar{\chi} \in \mathrm{L}(\bar{\lambda})$. It follows that $L$ is a closed mapping and $L$ is upper semicontinuous on $C^{* 0}$. From Lemma 4.5, we know that $\bar{X}=\bigcup_{\lambda \in C^{* 0}} \bar{X}_{\lambda}$ is connected.

Remark 4.7. Compared with results of $[16,17,23]$, Theorem 4.6 proposes linear scalarization method to obtain the connectedness of the solution set for (VEP) without the compactness or C-pseudomonotonicity which is different from the ones used in the previous literature.

Theorem 4.8. Assume that Assumption 3.1 holds, $\mathrm{h}$ is $\mathrm{C}$-pseudomonotone and $\mathrm{R}_{1}=\{0\}$. If $\mathrm{W}=\{\mathrm{h}(\mathrm{x}, \mathrm{y}): \mathrm{x}, \mathrm{y} \in$ $\mathrm{K}\}$ is bounded, then the solution set for (VEP) is connected.

Proof. It follows from Theorem 3.16 that coercivity condition (C) holds. Repeating the proof of Theorem 4.6, we obtain the results.

\section{Acknowledgment}

The authors are very indebted to the reviewers for their valuable comments and corrections which improved the original manuscript. This research is supported by the Natural Science Foundation of China (11671228, 61403228) and the Natural Science Foundation of Shandong Province (ZR2016AM10).

\section{References}

[1] L. Q. Anh, P. Q. Khanh, Semicontinuity of the solution set of parametric multivalued vector quasiequilibrium problems, J. Math. Anal. Appl., 294 (2004), 699-711. 1

[2] L. Q. Anh, P. Q. Khanh, D. T. M. Van, J.-C. Yao, Well-posedness for vector quasiequilibria, Taiwanese J. Math., 13 (2009), 713-737. 1, 4.1

[3] Q. H. Ansari, F. Flores-Bazán, Recession methods for generalized vector equilibrium problems, J. Math. Anal. Appl., 321 (2006), 132-146. 1

[4] J.-P. Aubin, I. Ekland, Applied Nonlinear Analysis, John Wiley \& Sons, New York, (1984). 4.5

[5] M. Bianchi, N. Hadjisavvas, S. Schaible, Vector equilibrium problems with generalized monotone bifunctions, J. Optim. Theory Appl. 92 (1997), 527-542. 2.2, 3.1, 3.2

[6] M. Bianchi, R. Pini, Coercivity conditions for equilibrium problems, J. Optim. Theory Appl., 124 (2005), 79-92. 1

[7] M. Bianchi, R. Pini, Sensitivity for parametric vector equilibria, Optimization, 55 (2006), 221-230. 1

[8] M. Bianchi, S. Schaible, Generalized monotone bifunctions and equilibrium problems, J. Optim. Theory Appl., 90 (1996), 31-43. 1, 3.1 
[9] G.-Y. Chen, X.-X. Huang, X.-Q. Yang, Vector Optimization and Set-Valued and Variational Analysis, Spinger, Berlin, (2006). 2.1, 2

[10] J.-H. Fan, R.-Y. Zhong, Stability analysis for variational inequality in reflexive Banach spaces, Nonlinear Anal., 69 (2008), 2566-2574. 2, 2.6, 4.2

[11] A. P. Farajzadeh, A. Amini-Harandi, On the generalized vector equilibrium problems, J. Math. Anal. Appl., 344 (2008), 999-1004. 1

[12] F. Flores-Bazán, Existence theorems for generalized noncoercive equilibrium problems: The quasiconvex case, SIAM J. Optim., 11 (2001), 675-690. 1, 3.1

[13] F. Flores-Bazán, Existence theory for finite-dimensional pseudomonotone equilibrium problems, Acta Appl. Math., 77 (2003), 249-297. 1, 3.1

[14] F. Flores-Bazán, F. Flores-Bazán, Vector equilibrium problems under asymptotic analysis, J. Global Optim., 26 (2003), 141-166. 1

[15] F. Giannessi, A. Maugeri, P. M. Pardalos, Equilibrium Problems: Nonsmooth Optimization and Variational inequality Models, Springer, New York, (2006). 1

[16] X. H. Gong, Efficiency and Henig efficiency for vector equilibrium problems, J. Optim. Theory Appl., 108 (2001), 139-154. $1,4.3,4.7$

[17] Y. Han, N.-J. Huang, The connectedness of the solutions set for generalized vector equilibrium problems, Optimization, 65 (2016), 357-367. 1, 4.3, 4.7

[18] R. Hu, Y.-P. Fang, On the nonemptiness and compactness of the solution sets for vector variational inequalities, Optimization, 59 (2010), 1107-1116. 1

[19] R. Hu, Y.-P. Fang, Strict feasibility and stable solvability of bifunction variational inequalities, Nonlinear Anal., 75 (2012), 331-340. 4.2

[20] X. X. Huang, Y. P. Fang, X. Q. Yang, Characterizing the nonemptiness and compactness of the solution set of a vector variational inequality by scalarization, J. Optim. Theory Appl. 162 (2014), 548-558. 1, 2, 3.8, 3.18

[21] I. V. Konnov, O. V. Pinyagina, A descent method with inexact linear search for nonsmooth equilibrium problems, Comp. Math. Math. Phys., 48 (2008), 1777-1783. 1

[22] S. J. Li, M. H. Li, Levitin-Polyak well-posedness of vector equilibrium problems, Math. Methods Oper. Res., 69 (2009), 125-140. 1, 4.1, 4.3

[23] Q.-Y. Liu, X.-J. Long, N.-J. Huang, Connectedness of the sets of weak efficient solutions for generalized vector equilibrium problems, Math. Slovaca, 62 (2012), 123-136. 1, 4.3, 4.7

[24] R. T. Rockafellar, R. J. Wets, Variational Analysis, Springer, Berlin, (1998). 2, 2.5

[25] I. Sadeqi, C. G. Alizadeh., Existence of solutions of generalized vector equilibrium problems in reflexive Banach spaces, Nonlinear Anal., 74 (2011), 2226-2234. 1

[26] M. Sofonea, Y.-B. Xiao, Fully history-dependent quasivariational inequalities in contact mechanics, Appl. Anal., 95 (2016), 2464-2484.

[27] G. Wang, Existence-stability theorems for strong vector set-valued equilibrium problems in reflexive Banach spaces, J. Inequal. Appl., 2015 (2015), 14 pages. 1, 4.2

[28] G. Wang, X. X. Huang, Levitin-Polyak well-posedness for optimization problems with generalized equilibrium constraints, J. Optim. Theory Appl., 153 (2012), 27-41. 1, 4.1

[29] G. Wang, X. X. Huang, J. Zhang, Levitin-Polyak well-posedness in generalized equilibrium problems with functional constraints, Pac. J. Optimi., 6 (2010), 441-453.

[30] Y.-M. Wang, Y.-B. Xiao, X. Wang, Y. J. Cho, Equivalence of well-posedness between systems of hemivariational inequalities and inclusion problems, J. Nonlinear. Sci. Appl., 9 (2016), 1178-1192. 1, 4.1

[31] Y.-B. Xiao, N.-J. Huang, Y. J. Cho, A class of generalized evolution variational inequalities in Banach space, Appl. Math. Lett., 25 (2012), 914-920. 1

[32] Y.-B. Xiao, N.-J. Huang, M.-M. Wong, Well-posedness of hemivariational inequalities and inclusion problems, Taiwanese J. Math., 15, (2011), 1261-1276. 1, 4.1

[33] L. C. Zeng, J. C. Yao, Modified combined relaxation method for general monotone equilibrium problems in Hilbert spaces, J. Optim. Theory Appl., 131 (2006), 469-483. 1

[34] L. Zhang, S.-Y. Wu, An algorithm based on the generalized D-gap function for equilibrium problems, J. Comput. Appl. Math., 231 (2009), 403-411. 1 